\title{
FORMAL SUPPORT METHODS IN DESIGN: DISCUSSION
}

\author{
STEPHAN RUDOLPH \\ Stuttgart University, Germany
}

\section{General Considerations}

Computers have affected many areas of our lives since their first appearance. The changes induced by computers have not only affected our way of doing things or monitoring and controlling processes. Computers have also changed our perception of the world and the way we think. For this reason the existence of computers as modern production means has had a significant influence on our way of doing science and design research as well. The modern paradigm "design is search" versus the paradigm of the former century "design is composition" accounts for this change of views and methods of many engineering design researchers. Computers have become the medium of choice of many.

However, despite the widespread applications of computers in virtually every area of engineering and design, little importance has been attached to fundamental, not so obvious but nevertheless important, implications of computer usage. A closer look at these fundamental implications of computer usage in general and especially in the area of engineering design reveals the following three main issues.

1. Representation. In order to write a useful program, a set of operands and operators needs to be specified. They are intended to be representations of real objects and the permissible manipulations on these. As with any representation, the question occurs as to what extent this representation is complete, and, since all operations occur on these operands and are thus limited to these, whether the underlying closed-world assumption is valid.

2. Mapping. Computer programs generally compute a certain set of output data $O$ from a certain set of input data $I$. Mathematically speaking, a computer program represents an algorithm $P$ which maps the input data onto the output data, thus $P: I \longrightarrow O$. However, the set of all algorithms $P$ is a true subset of all mathematically imaginable mappings $M$, thus $P \subset M$. Computers can therefore in general only deal with problems which are algorithmically solv- 
able. This is a major restriction on the kinds of problems that computers are capable of dealing with.

3. Reasoning. Formal models of logic rely heavily on certain characteristic properties such as proofs of consistency, completeness or correctness. However, any extension of theoretical models is only feasible when through comparison of the natural world with the model predictions discrepancies are identified by observations or experiments. Because of the continuing lack of devices serving as computer interfaces suited for such needs, these inconsistencies cannot be detected and conceptualized automatically. This seems, at least for the near future, to remain a domain for the problem identification and problem solving capabilities of humans.

The first and second of these three main issues are today more or less well identified and understood. They have been encountered and tackled since the appearance of the first implementations of numerical algorithms in computer programs. Take, for example, the consequences of the finiteness of the representation of numerical values of program objects, i.e. of the program variables, are all kinds of stability and convergence problems in numerical algorithms and originated much research devoted especially to the solution of these problems. Today it is generally accepted that the fact of doing computation on machines with finite precision, i.e. incomplete representation, lies at the heart of many problems and the trend to symbolic computation can be interpreted as one way to try to circumvent this problem at the expense of the introduction of other difficulties.

The third major issue is of a more difficult nature and not so obvious. For this reason it is investigated here in some more detail. The continuing and correct execution of a set of two operations is studied in Figure 1. In this figure, the outcome of a surface walk of a person (the object) to which two different operations can be applied (walking infinitesimally 'straight' for a certain amount of time and then making a ninety degree left turn), is drawn. As shown in the left hand part of Figure 1, going 'straight' on the surface of some (flat) plane from a point $A$ to a point $B$, making a ninety-degree turn at $B$, going then 'straight' from point $B$ to a point $C$, making a ninety degree turn at $C$, going on the surface a third time 'straight'
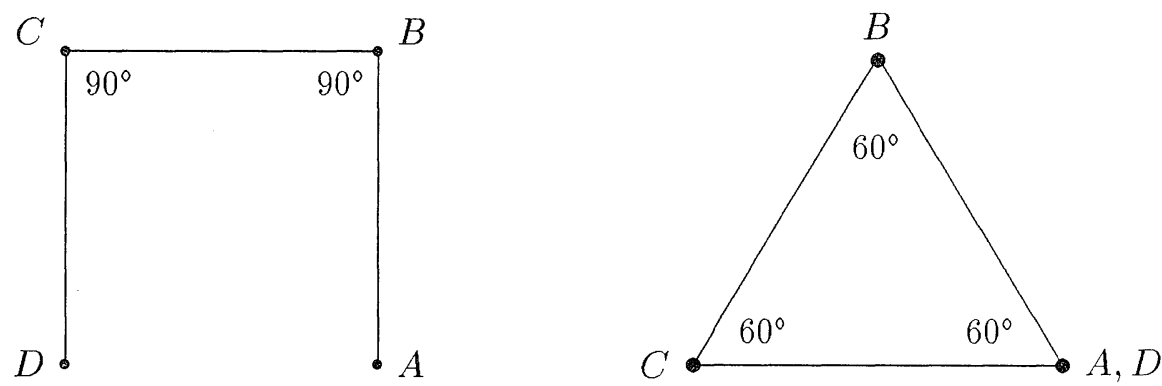

Figure 1. Two walks on a 2-dimensional flat surface 
from point $C$ leads finally to point $D$, which is (expected to be) quite distinct from the point $A$ of departure. If however the point $D$ turned out to be identical to $A$, one realizes that there is something wrong - this can only happen when the angles are sixty and not ninety degrees, as shown in the right hand side of Figure 1.

However, it is also imaginable that another reason lies at the origin of the difference in the expected outcome of the surface walk. This is shown in Figure 2, where the surface walk happens to take place not on a 2-dimensional plane, but on the surface of a 3-dimensional sphere. There it is easily possible to arrive at point $A$ again, after walking 'straight' three times and making ninety degree turns at $B$ and $C$ respectively. Contrary to humans, computers cannot 'experience' such discrepancies in computer program simulations, since the concept of the dimensionality of the surface has to be a part of the a priori chosen representation defined by the programmer. Since experience is inseparably tied to reality, while simulation depends

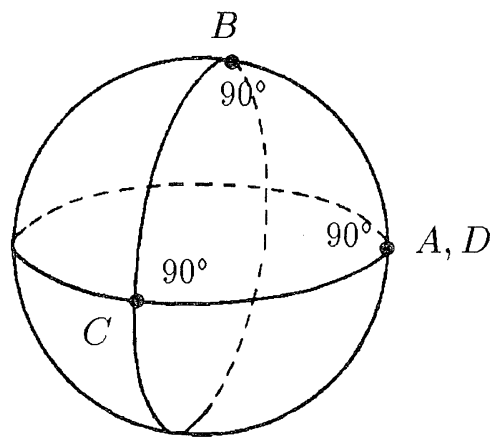

Figure 2. A walk on a 3-dimensional sphere

on a representation of the latter, it is important to realize that both the experience of 'inconsistency' as a result after executing a set of 'correct' operations, as well as an attempt to formalize the occurrence of such an inconsistency into an extension of the currently valid (geometrical) theory to a more general theory, seems very hard if not even impossible to automate. A general theory, which does explain the new experiences, has to contain and explain all former experimental knowledge as special cases as well. It is strongly questioned whether computers are generally capable of doing such truly conceptual generalizations. If computers were able to, then one could argue that one could create a program which could become monotonically 'smarter', just by executing itself. Since all the operations inside a program rely on the inbuilt representations provided a priori by the programmer, in reality the underlying metric of the space does change automatically and without notice, since it depends only on the individual problem area under consideration.

While these considerations might seem pointless or even superficial to many, the strong connection of these thoughts to the superior capabilities of humans over computers to form novel theories and to be innovative in an engineering sense 
should be evident. These are capabilities which do heavily rely on a personal experience of 'inconsistency', which can be strongly subjective and does not even animate two different humans necessarily to come to the same conclusions. Experience shows that 'inconsistency' can pass unperceived and it is possibly just this which makes true innovation such a rare event.

While keeping these principles and fairly general implications of the use of computers in mind, the next section investigates in more detail the various specific effects of the use of computers in engineering design today.

\section{Computers in Engineering Design}

Besides the general theoretical implications of computer usage discussed in the previous section, the existence of computers has also affected the practice of doing science and engineering design research. In former times science seemed to be in principle an iterative sequence of empirical and careful observation of natural phenomena, its modeling and then a continuing comparison of the model predictions with further observations. Today, the focus is often shifted to a methodological sequence of the definition of axioms and the specification of a set of permitted operands and operations (i.e. a formal logic based on the axioms), which is then used to derive a set of statements as formally derivable consequences of a certain sequence of operations.

While on the one hand this approach offers the advantage of being formal and thus able to be objectified and programmable, there is on the other hand also the danger of losing the vital link between the formal (design) model axioms and the reality of the real (design) world. At the worst this may even lead to clean, nice and powerful formal (design) models and methods, which have nothing to do anymore with the real (design) world.

This motivates the question how the usefulness of newly suggested design models or methods can be evaluated. Two possibilities were discussed without agreed conclusions.

1. Benchmarking. Arguments in favor of benchmaking included the transparency of the evaluation process through a clear statement of the performance criteria, and the need for such a procedure for comparison of newer design methodologies with older ones. Arguments against benchmarking included the impossibility of stating generally valid performance criteria. It was argued that a particular design solution may not be determined so much by the choice of a certain design procedure (i.e. the design methodology). as by the existence of problem specific boundary conditions. By their nature, these boundary conditions do largely differ from case to case. Additionally, there might be an unconscious tendency of humans to evaluate and compare the outcome only, i.e. the design solutions, and not the differences in the design process 
itself as a consequence of another axiomatic foundation of the used design methodology.

2. Problem Collections. As an alternative to the preceding point, where it turned out that benchmarking of design methods seems difficult to carry out, the use of design cases as case studies was suggested to help the comparison of the different aspects and procedural steps of the various design methods with one another. However, it was argued that it would be very difficult, if not impossible, to establish such a required reference collection of meaningful design examples.

While the discussers felt that benchmarking and case studies are valid tests in many other areas of engineering, its application to engineering design would be advantageous to increase transparency and objectivity in the field, if the above mentioned difficulties could be overcome. As a first step in this direction, it was suggested to look at the collection of the different design methods as a toolbox, where each tool might be ideally suited for its specific purpose. On the other hand, the very same tool, ideally suited for a specific purpose, may perform only adequately or even badly for other purposes outside its original scope.

The previous arguments show that it is important to characterize the precise scope and applicability of any newly developed design theory or method. The various theoretical and practical limitations of the method used should also be clearly identified. The set of all critical underlying assumptions of the method should also be explicitly stated. A thorough treatment of these theoretical and practical issues is not only a question of individual scientific honesty and the personal working style of any researcher, but is essential for any true scientific advancement in the field of engineering design. It also assists the necessary characterization of the various design methods and methodologies by stressing their individual differences versus their commonalities in the above sense.

The other important point in the establishment of computational theories of design seems to be the nature and the choice of a set of appropriate axioms. Since any statement formally deduced in such a logical framework can be shown to be based on the previously chosen set of axioms, the question arises how much validity and unforseeable consequences are inherent to the choice of a certain set of axioms. Is their choice really free and arbitrary? From a purely theoretical point of view the answer is: Yes, of course! The main concern of theory building is the formal deduction of statements using some sort of established logic.

On the contrary, from an epistemological viewpoint, the answer to the very same question is: No, of course not! In thermodynamics for example, it is always observed that the heat flows from the hotter object to the colder one, and never in the reverse direction. If the empirical observation of such a unique behavior had not been made, this principle could not have been established. Thus, the second principle of thermodynamics is not arbitrarily 'defined', but is in fact completely epistemologically based and justified. It is just this epistemological basis of the 
axioms which is the vital link of the established theoretical model to reality and provides in large part the validity of all the subsequent formal deductions by means of this theory.

What, then, are the observations in every day design reality which could be used as an epistemological basis of design and formulated as its axiomatic foundation? This is a tough question to answer. For the moment, there seems to exist no common agreement in the engineering design community on such an axiomatic foundation in the area of engineering design. There are many existing paradigms in the form of Design for ' $X$ ', i.e.

- Design for Manufacturing,

- Design for Assembly,

- Design for Disassembly,

- Design for Recycling, etc.,

which do summarize and characterize some basic observations about the design process. However, the above paradigms are by no means formal necessities in a mathematical sense. For this reason, they may never qualify for a true axiomatic foundation of engineering design from an epistemological viewpoint. It seems that this fact of a still unidentified epistemological foundation of engineering design represents one of the most important current bottlenecks of design research. Any significant contribution in this respect would be an important theoretical contributions to the development of a future science of engineering design sought by many.

If the identification of an epistemologically justified axiomatic foundation of a theory is such a difficult thing to do, what general heuristics do mathematicians and scientists use when they have developed a new theory and try to judge its usefulness? In such a case one may resort to one or more of the following criteria: Is the theory

- powerful?

- simple?

- elegant?

- beautiful?

In fact, these criteria reflect the formal embedding of logic in aesthetic and of aesthetic in ethic. This means that the existence and the outcome of the former can be explained and justified by the latter. This philosophical view refers to ancient Greek philosophy and dates back to Aristotle. A sketch of this mutual embedding of logic in aesthetics and of aesthetics in ethics is shown in Figure 3.

So why are computers, despite the drawbacks discussed, such attractive research and application tools? The answer seems to be obvious. Once a software program exists, the program and its data can easily be duplicated, distributed and shared with others. Once programmed, the high execution speed of relatively simple operations provides an alternate way to explore possibilities and simulate the behavior of objects which have never existed in reality. Search techniques, the paradigm 


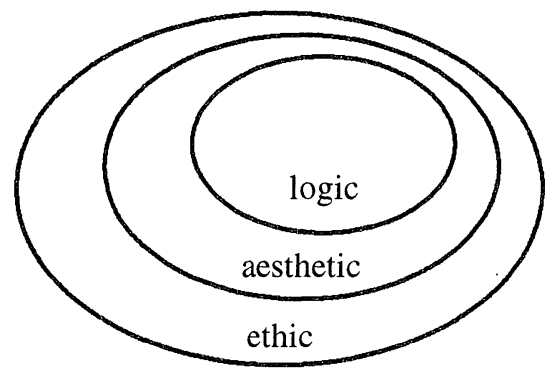

Figure 3. Aristotlean philosophy

"design is search" and simulation program packages using finite element techniques are well known and accepted examples of this.

The following two papers of this workshop session show besides their individual attempts to contribute to the advancement of the field, examples of individual compromises in terms of the above issues as well. The discussion of the two papers after their presentation during the workshop session is summarised below.

\section{Paper Discussion}

The paper Formal Concept Analysis in Design by Mihaly Lenart describes the use of Hasse diagrams for the analysis of complex situations in order to facilitate decision making in design. Based on a matrix containing the relations between the design objects and their properties, a Hasse diagram is constructed founded on the theoretical concept of lattices.

Possible benefits of such a purely formal approach are the apparent generality of the method and its independence of a particular problem area. The Hasse diagrams provide a transparency of the coupling between objects and properties. The representation of this coupling also allows the identification of a hierarchical order. The representation form can be nested to hide details and to deal with a large number of edges and vertices. In terms of computational complexity, an exponential growth is expected on average, but nesting can be used to reduce the number of displayed edges and vertices.

Possible disadvantages of the method are the missing representation of design contexts in this context free method. Also, due to the lack of familiarity of most designers with theoretical concepts such as lattices and Hasse diagrams, the two questions of the adequacy of the method concerning the requirements in engineering design and of the demonstration of the practical usefulness of the theoretical approach, could not be answered.

As a future perspective, the author was encouraged to demonstrate the suggested technique using more realistic engineering design examples and to clarify 
the discussion of the usefulness and limitations of his approach to design analysis and/or design synthesis.

It was interesting to see that strong formal methods can sometimes also suffer from their formality when applied. Abstract concepts, like the theoretical concept of lattices, make it sometimes more difficult to convince prospective users to make the required extra effort to become familiar with a particular mathematical technique. Due to the 'inertia' inherent in many humans, new techniques are often only adopted when they can handle tough cases and solve important problems other techniques have been shown repeatedly to be incapable of solving. This seems to be one of the major obstacles for many new methods and represents one of the more important factors slowing down the adoption of methodological change and innovation.

In contrast to the formal theoretical concept of lattices, the second paper of this session relied on the known and already well established systematic German engineering design approach developed by Beitz, Ehrlenspiel, Hubka, Koller, Pahl, Roth and others.

The paper Supporting the Design Process by an Integrated Knowledge-Based Design System by Hans Grabowski, Ralf-Stefan Lossack and Clemens Weis describes the embedding of the German design methodology into a framework of a knowledge-based CAD system. German design methodology considers the design process as an iterative process of concretization performed at different levels of abstraction. These different levels of abstraction are classified into the following.

1. Requirements modeling layer, containing the preconditions of the design, the definition of the product requirements and the description of the product's immanent task structure.

2. Functional modeling layer, serving to represent the functions and the functional interrelationships of the design objects to be developed.

3. Conceptual modeling layer, containing all the information to describe the solution concept of a design, such as physical solution principles, effective spaces and the grouping of the functional structure into a conceptual structure.

4. Shape modeling layer, completing the above product modeling layers by the geometrical definitions to fully define the 3-dimensional parts with assigned material properties and their combination into a part group structure.

In each of the above modeling layers, the following activities can be performed to advance the current solution to the next solution state. These are the steps to

- detail

- vary

- concretize

- combine or

- abstract

the solution state. 
Starting with this description of the major elements of the German design methodology and the classification into the above modeling layers, the possible future integration and support of this phase oriented view of the design process by means of a knowledge-based CAD system was described. It was suggested this methodology be provided with a conceptual specification of how to navigate through the design process using a dynamic process model. The complete envisioned approach, including the still missing knowledge based modules for some of the four modeling layers, was demonstrated using the example of a more and more detailed design of a robot gripper. A prototype of this system is already partially implemented as a working software tool.

Possible benefits of such a systematic design method lie in the systematic decomposition of the complex design process, which helps to suppress hidden assumptions. Further, the systematic investigation and use of physical principles might help to guide the search for novel design solutions. This is a similar procedure to the morphological box approach, where physical principles are systematically combined to create new conceptual design solutions. Finally, the idea to support the designer by means of case-based knowledge to help the navigation through a dynamic design process model seems to be promising.

Possible limitations of the method might be its restriction to specific problem areas where functional modeling using physical principles is applicable. Also, the necessity to specify the definition of the input/outputproperties of the future design object might be difficult in some complex design cases.

\section{General Outlook}

Design objects are a part of our daily environment. In this respect, design objects have many consequences for humans. One could even say that design objects and thus design affects society: the invention of the automobile has transformed our society in the last one hundred years significantly. The existence of cars has created the need for roads, highways and parking lots. This affected cities as well as the shape of our countrysides. Even the organization of our cities is affected by the consequences of the ability to be readily mobile with personal cars.

Since most events are more likely to be coupled than isolated events from one another, one can say that society affects design at least as much as design affects society. The creation of design 'styles' and the existence of laws, design norms and expressions like 'political correctness' show the closure of this feed-back loop.

Additionally, the growing competition in world wide consumer markets increases the pressure on virtually every company towards new, modern and more innovative products. Besides the two aspects of innovation in engineering designs, e.g. product-driven designs and technology-driven designs, what is the true source of innovation? Is there one common concept of it? Can innovation be algorithmically formulated or enforced? These are some of the fundamental and driving 
questions of engineering design research today which make it such a challenging field with possible benefits to many other scientific disciplines. Determining the theoretical issues of the possibilities and the impossibilities of computer support in engineering design has therefore been one of the many interesting topics of this workshop session. However, remarkably many final answers to this problem of humankind still have to be searched for. 